\title{
Alkaline niobate nanowires as opto-mechanical probes
}

\author{
Fabrizia Dutto*a ${ }^{\text {a }}$ Aleksandra Radenovic ${ }^{\mathrm{a}}$ \\ ${ }^{a}$ Laboratory of Nanoscale Biology, Institute of Bioengineering, School of Engineering, EPFL, 1015 \\ Lausanne, École Polytechnique Fédérale de Lausanne (EPFL), CH-1015 Lausanne, Switzerland
}

\begin{abstract}
Perovskite alkaline niobate $\left(\mathrm{XNbO}_{3}\right)$ nanowires are attracting lots of attention having a variety of interesting properties such as significant nonlinear optical response, pronounced birefringence, considerable piezoelectric, pyroelectric, photorefractive, and photocatalytic response, as well as superior mechanical and chemical stability. Their ability to efficiently generate second harmonic signals (SHG) and their birefringence allow the use of these nanostructures as local mechano-optical probes for single molecule detection.

To assess which type of nanowires is suitable for specific application, we performed a comparative study on the nonlinear optical response of the different types of chemically synthesized alkaline niobate nanowires: sodium niobate $\left(\mathrm{NaNbO}_{3}\right)$, potassium niobate $\left(\mathrm{KNbO}_{3}\right)$ and lithium niobate $\left(\mathrm{LiNbO}_{3}\right)$ nanowires. An optical trap setup has been used to demonstrate the possibility to steadily trap the nanowires, their ability to generate high second harmonic signals, to waveguide this signal and to be rotated under a highly focused laser beam with changing polarization. Different applications are suggested for the three materials, such as $\mathrm{LiNbO}_{3}$ nanowires as imaging markers, while $\mathrm{KNbO}_{3}$ and $\mathrm{NaNbO}_{3}$ nanowires for trapping and torque experiments and $\mathrm{NaNbO}_{3}$ nanowires to waveguide SHG light. Functionalization of the $\mathrm{XNbO}_{3}$ nanowires has been studied and successfully implemented. This is a first crucial step toward their use in biomedical imaging and single molecule applications.
\end{abstract}

Keywords: alkaline niobate nanowires, $\mathrm{NaNbO}_{3} \mathrm{KNbO}_{3} \mathrm{LiNbO}_{3}$, chemical synthesis, optical trapping, SHG, waveguiding, torque

\section{INTRODUCTION}

All alkaline niobates are noncentrosymmetric crystalline dielectric materials with very promising and interesting properties and characteristics. Noncentrosymmetric crystals are of particular interest because of their symmetrydependent properties that are the basis of numerous applications ${ }^{[1]}$, such as second-order nonlinear optical (NLO) behavior and birefringence. These properties can be exploited for single molecule techniques based on second harmonic generation (SHG) or on torque application and detection.

The fabrication of alkaline niobates in form of nanowires have been achieved through either hydrothermal synthesis process, for $\mathrm{NaNbO}_{3}$ and $\mathrm{KNbO}_{3}$ nanowires ${ }^{[2-6]}$, or molten salt synthesis one, for $\mathrm{LiNbO}_{3}$ nanowires ${ }^{[7]}$. The overall performance of the three types of wires is compared: by combining the obtained objects with our optical trap, nanowires can be trapped, manipulated, rotated and their emission of a SHG signal can detected.

\section{PRINCIPLE OF THE SYSTEM}

\subsection{Second Harmonic Generation (SHG) principle}

Materials lacking inversion symmetry, as noncentrosymmetric crystals or solid surfaces where the symmetry is broken by the presence of an interface, can exhibit non linearity ${ }^{[8]}$. Alkaline niobates $\left(\mathrm{XNbO}_{3}\right)$, as noncentrosymmetric material, are able to generate a strong second harmonic signal.

SHG is a process first demonstrated by Franken et al. ${ }^{[9]}$ in 1961 exploiting the invention of the laser: the high intensity field of a laser beam creates a field inside the lighted material comparable with the incident one, bringing a modification of the optical properties of a material called non linear properties. The description of this phenomenon can be done by expressing the polarization $\mathbf{P}$ as a power series of the field strength $\mathbf{E}$ as

$$
\mathbf{P}(t)=\varepsilon_{0}\left(\chi^{(1)} \mathbf{E}+\chi^{(2)} \mathbf{E} \mathbf{E}+\chi^{(3)} \mathbf{E} \mathbf{E} \mathbf{E}+\ldots\right)
$$

where $\varepsilon_{0}$ is the permittivity of free space, $\chi^{(1)}$ is the linear susceptibility, $\chi^{(2)}$ and $\chi^{(3)}$ are the second and third order non linear optical susceptibilities respectively ${ }^{[8]}$.

*fabrizia.dutto@epfl.ch; phone: 0041 (0)21 6931162

Optical Trapping and Optical Micromanipulation IX, edited by Kishan Dholakia, Gabriel C. Spalding, Proc. of SPIE Vol. 8458, 84581X · @ 2012 SPIE · CCC code: 0277-786/12/\$18 - doi: 10.1117/12.929466 
The second order susceptibility is a third rank tensor that is associated to the SHG phenomenon, in simple terms explained as the interaction of a non linear material with two photons (with the same frequency $\omega$ ), resulting in the creation of a photon with a double frequency $2 \omega$. The SHG signal is stable over time and do not bleach. Moreover the process is nonresonant thus the SHG can be excited with any frequency.

By trapping a non linear object with optical tweezers, the laser beam forming the trap simultaneously causes the generation of a second harmonic signal at half the impinging wavelength. The tunability of the process allows the use of different wavelengths for SHG excitation and samples characterization.

\subsection{Optical tweezers and optical torque wrench}

Optical tweezers are an important tool that in recent years has successfully been employed in many single molecule biophysics applications ${ }^{[10]}$. The system is based on tightly focusing a laser beam with an objective of high numerical aperture (NA). The common trapping technique allows the linear manipulation of nanometer and micrometer sized dielectric particles and force measurement. The electric field gradient forming the narrowest point of the focused beam, attracts particles towards the center of the beam.

Recent improvements in the field extend the optical tweezers functionality to the possibility of rotate particles and measure their torque. The principle on which both approaches are based is the same: a transfer of momentum, linear or angular, from the trapping beam to the particle, due to scattering of incident photons, leads to the production of optical forces in the former case and optical torque in the latter one.

Light can carry angular momentum (AM) in two distinct forms: spin angular momentum, associated with the polarization state of the beam and orbital angular momentum, associated with the spatial distribution of the beam. In our application the torque is produced by changing the polarization of the light, thus by altering the spin AM.

Torque can be transferred either by absorption or, to avoid heating, to a nonabsorbing particle able to alter the polarization of the incident beam. The second case is fulfilled by particles that present an external (shape birefringence) or an internal (natural birefringence) anisotropy ${ }^{[11]}$ thus a non uniform spatial distribution of properties. Birefringent objects present different indices of refraction depending on the orientation of the electric field vector relative to the optic axis of the crystal. As a consequence, the interaction mechanism of the beam with the object is dependent on the orientation of the crystal with respect to the incident light angle. Whenever the light is not entering the object parallel to the optical axis of the crystal, a phase shift between the different components of the beam will be induced, altering the polarization state of the incident light. For the conservation of the AM, the torque acting on the trapped particle is equal and opposite to the change in the AM. The torque can thus be expressed as the modification of the light polarization state of the trapping beam as it passes through the particle.

Many anisotropic crystals are available in nature such as quartz, calcite, niobates, ... By impinging onto the trapped nanowire with either a left or a right-handed circular polarization (CP) beam, an angular momentum $\pm \hbar$ and an energy $\hbar \omega_{0}$ per photon (where $\hbar$ is the reduced Plank constant and $\omega_{0}$ is the optical angular frequency of the beam) is transferred to the particle. A transfer of AM to the particle is also possible by constantly rotating a linearly polarized (LP) beam, that, by itself, do not contain a net angular momentum cause the left and right circular components are equal. In both cases the interaction of the beam with the nanowire creates an imbalance of power of left and right circular components $\left(\mathrm{P}_{\mathrm{L}}\right.$ and $\left.\mathrm{P}_{\mathrm{R}}\right)$ in the transmitted beam. By monitoring the imbalance of left and right circular components in the transmitted beam, a direct measure of the torque $\tau$ is possible ${ }^{[11-19]}$ :

$$
\tau=\frac{P \Delta \sigma}{\omega_{0}}
$$

where $\mathrm{P}$ is the total power of the beam, $\sigma$ is the degree of circular polarization defined as $\sigma=\left(P_{R}-P_{L}\right) / P$ and $\Delta \sigma$ is the change in the degree of circular polarization defined as $\Delta \sigma=\sigma_{\text {final }}-\sigma_{\text {initial }}$.

\section{METHODS}

\subsection{Chemical synthesis}

All three types of $\mathrm{XNbO}_{3}$ nanowires have been fabricated by chemical synthesis, either hydrothermal or molten salt one, employing chemicals commercially available (Sigma-Aldrich) without any further purification.

Hydrothermal synthesis is a technique used to crystallize substances under high temperatures and high pressures. Thanks to the use of an autoclave, a thick-walled steel cylinder with a hermetic seal, crystalline materials can be obtained in one step. The large amount of material that can be fabricated, the simplicity and the speed of the synthesis make this 
approach very convenient. To optimize synthesis parameters one can adjust temperature, time, pressure (by external pressure or degree of filling of the autoclave), caustic soda concentration, solid-liquid ratio and additives. In this way it is possible to control particle size and morphology, switching from the production of unspecified clusters to cubes or wires.
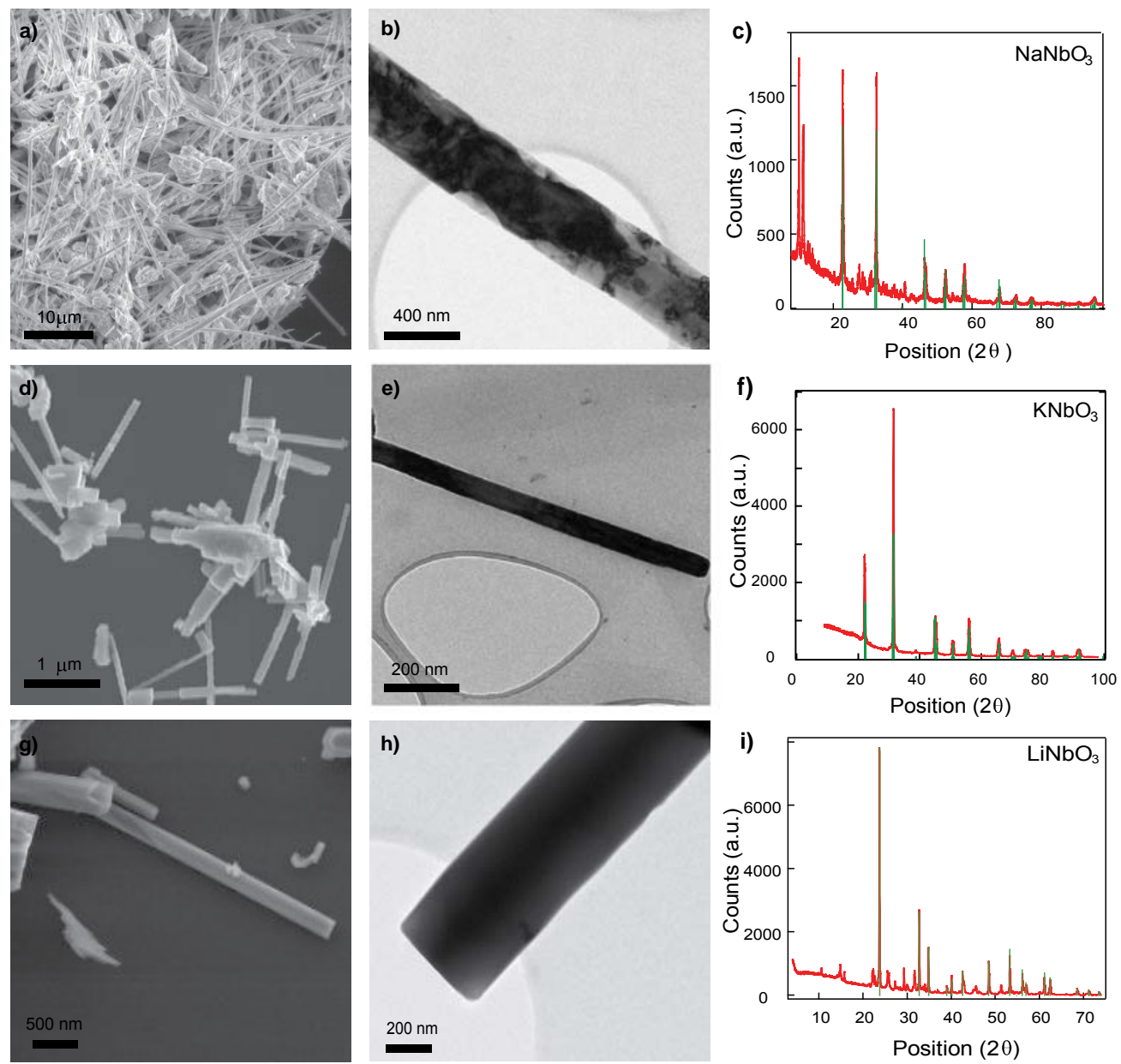

Figure 1: a) SEM image of hydrothermally synthetized $\mathrm{NaNbO}_{3}$ nanowires. b) TEM image of a hydrothermally synthesized $\mathrm{NaNbO}_{3}$ nanowire. c) $\mathrm{NaNbO}_{3}$ XRD measurement (red) and theoretical peaks (green). d) SEM image of hydrothermally synthesized $\mathrm{KNbO}_{3}$ nanowires. e) TEM image of a hydrothermally synthesized $\mathrm{KNbO}_{3}$ nanowire. f) $\mathrm{KNbO}_{3} \mathrm{XRD}$ measurement (red) and theoretical peaks (green). g) SEM image of $\mathrm{LiNbO}_{3}$ nanowires made by molten salt synthesis. h) TEM image of a $\mathrm{LiNbO}_{3}$ nanowire made by molten salt synthesis. i) $\mathrm{LiNbO}_{3}$ XRD measurement (red) and theoretical peaks (green).

Polycrystallined $\mathrm{NaNbO}_{3}$ nanowires, with an aspect ratio up to 50 (see Figure 1a), have been produced hydrothermically by following the recipe proposed by Zhu et al. ${ }^{[3]}$. The employed procedure is the following: $0.25 \mathrm{~g}$ of $\mathrm{Nb} 2 \mathrm{O} 5$ is dispersed in a $15.33 \mathrm{ml}$ aqueous solution of $10 \mathrm{M} \mathrm{NaOH}$. The obtained solution is stirred for one hour at $50^{\circ} \mathrm{C}$, then sealed into a $23 \mathrm{ml}$ teflon-lined autoclave and subsequently heat for $2 \mathrm{~h}$ at $180^{\circ} \mathrm{C}$. The white precipitate is recovered by centrifugation, it is washed with ethanol and deionized water and then dried at $110^{\circ} \mathrm{C}$ overnight. By SEM characterization it is clear that most of the product has a nanowire shape (see Figure 1a). However, by checking the phase of the product by X-ray diffraction (XRD), a very small amount of $\mathrm{NaNbO}_{3}$ compound is detected, while most of the product $(85 \%)$ is a different phase of niobate such as heptasodium oxonium 19-oxohexaniobate 14-hydrate, $\mathrm{Na}_{7}\left(\mathrm{H}_{3} \mathrm{O}\right)\left(\mathrm{Nb}_{6} \mathrm{O}_{19}\right)\left(\mathrm{H}_{2} \mathrm{O}\right)_{14}$. A calcination step (annealing at high temperatures such as $550^{\circ} \mathrm{C}$ ), as suggested by Shi et al. [2], increases drastically the amount of $\mathrm{NaNbO}_{3}$ phase (up to $64 \%$ ).

Single crystalline $\mathrm{KNbO}_{3}$ nanowires with an aspect ratio around 20 (see Figure 1d) have been produced by following the recipe described by Wang et al. ${ }^{[20,21]}$. The process is very similar to the one implemented for $\mathrm{NaNbO}_{3}: 0.098 \mathrm{~g}$ of 
$\mathrm{Nb}_{2} \mathrm{O}_{5}$ is dispersed in a $3.68 \mathrm{ml}$ aqueous solution of $16 \mathrm{M} \mathrm{KOH}$. The addition of a surfactant, sodium dodecyl sulfate (SDS), into the solution influences the nucleation and the growth of nanocrystal, promoting an oriented aggregation of them into nanowires. The optimal concentration of SDS is $0.0025 \mathrm{~mol}^{[20,21]}$. After the usual stirring, the product is sealed into a $23 \mathrm{ml}$ teflon-lined autoclave and then heated for $24 \mathrm{~h}$ at $180^{\circ} \mathrm{C}$. XRD characterization confirmed that nothing but $\mathrm{KNbO}_{3}$ was formed into two crystalline phases: $38 \%$ of cubic phase and $62 \%$ of orthorhombical one.

Molten salt synthesis (MSS) had to be implemented for LiNbO3 nanowires synthesis since the hydrothermal approach wasn't successful. In this approach oxides corresponding to a perovskite compound are mixed with one or two salt species and then heated at a temperature above the melting point of the salt to form a flux of the salt composition. At this temperature the oxides are rearranged and then they diffuse rapidly into a liquid state of the salt. With further heating, particles of the perovskite phase are formed through the nucleation and growth processes.

By following the recipe reported by Santulli et al. ${ }^{[4]}$, single crystalline $\mathrm{LiNbO}_{3}$ nanowires can be fabricated with an average aspect ratio of 10 (see Figure $1 \mathrm{~g}$ ): $0.66 \mathrm{~g}$ of $\mathrm{Nb}_{2} \mathrm{O}_{5}$ is mixed to $1.86 \mathrm{~g}$ of $\mathrm{KCl}$ in a 1:10 ethanol solution. The mixture is dried at $80^{\circ} \mathrm{C}$ and then heated at $800^{\circ} \mathrm{C}$ for $3 \mathrm{~h}$. After several washing steps the obtained product is $\mathrm{KNb}_{3} \mathrm{O}_{8}$ in a nanowires form. By adding $400 \mathrm{ml}$ of $2 \mathrm{M} \mathrm{HNO}_{3}$ and by stirring the product for $48 \mathrm{~h}$, there is a conversion of $\mathrm{KN}_{3} \mathrm{O}_{8}$ nanowires into $\mathrm{H}_{3} \mathrm{ONb}_{3} \mathrm{O}_{8}$ ones. By annealing at $550^{\circ} \mathrm{C}$ for $1 \mathrm{~h}$ the nanowires are transformed into $\mathrm{Nb}_{2} \mathrm{O}_{5}$ ones. $\mathrm{LiNbO}_{3}$ nanowires can be finally obtained by adding $\mathrm{Li}_{2} \mathrm{CO}_{3}$ in $1: 1$ molar ration, by mixing it to an equal weight of $\mathrm{KCl}$ and by heating the product at $780^{\circ} \mathrm{C}$ for $10 \mathrm{~min}$. XRD characterization reveals the presence of $75 \%$ of trigonal $\mathrm{LiNbO}_{3}$ phase.

\subsection{Setup description}

The optical setup employed in this study is based on the use of a diode-pumped $\mathrm{Nd}-\mathrm{YVO}_{4}$ solid state laser (COMPASS 1064, 2.5W continuous wave, linear polarization, $\lambda=1064 \mathrm{~nm}$ ) with a $0.4 \mathrm{~mm}$ diameter collimated Gaussian beam as trapping beam. The beam is send through a polarizer (Thorlabs) and is passing afterwards through a power control system.

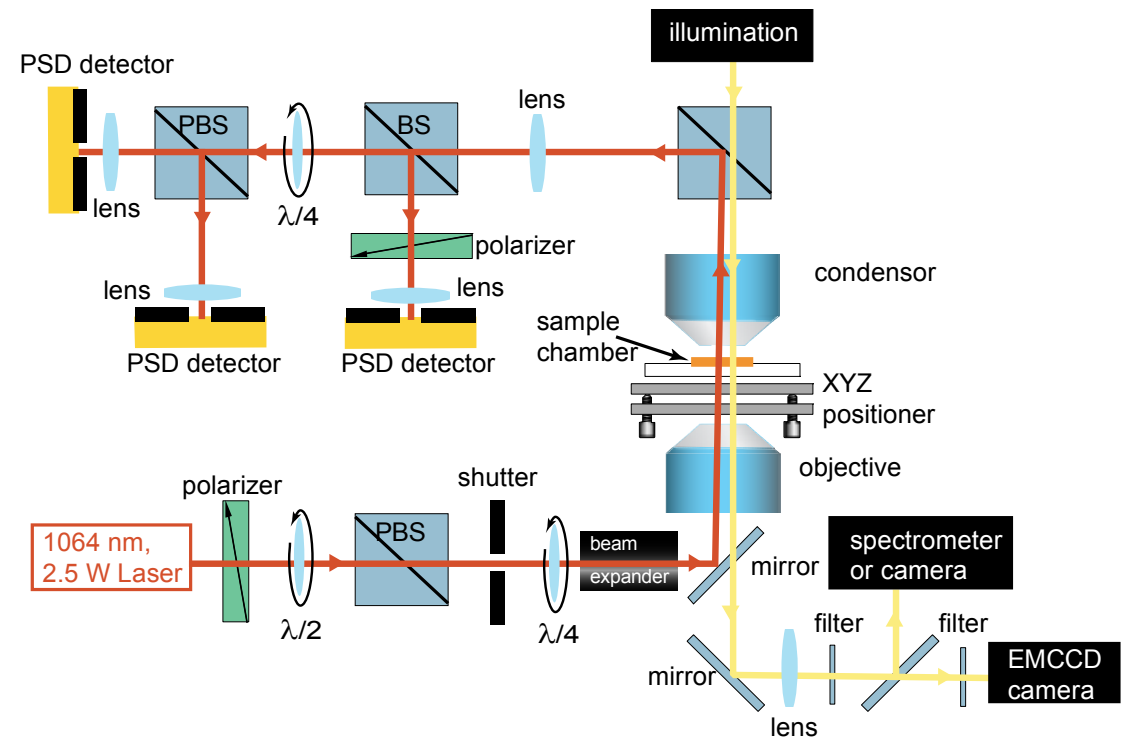

Figure 2: Schematic of the optical trap setup adapted for torque application and detection.

This system allows the use of the laser beam always at its maximum power, at which the intrinsic noise level is the minimum $(<1 \% \mathrm{rms}$ ), and the adjustment of the trap power by a half waveplate (HWP; Thorlabs) combined with a polarizing beam splitter (PBS; Thorlabs). In fact, by changing the half waveplate axis direction, the amount of power transmitted by the PBS is varying. After leaving the power control system, the trapping beam encounters a quarter waveplate (QWP; Thorlabs) used to control the polarization of the laser. By placing the QWP axis at $45^{\circ}$ respect to the beam linear polarization direction, the beam becomes circularly polarized. The birefringent particle interacting with it is 
then experiencing an angular momentum that results in its rotation. If the QWP axis is placed at $0^{\circ}$ the laser beam is not modified by the waveplate, thus keeps its polarization linear and in the same direction.

The beam is then expanded by a telescope $(5 \mathrm{x})$ to overfill the back aperture of the high numerical aperture (NA) water immersion objective (Nikon, 60x, 1.2 NA). This objective tightly focuses the beam to form the optical trap. A dichroic mirror is placed at $45^{\circ}$ after the telescope to deflect the laser beam into the objective and to transmit the illumination beam toward the cameras for imaging purposes. Trapped objects are imaged with the objective onto an Andor Ixon+ (DU987) camera that allows single photon detection and thus facilitates the low SH signal detection.

A custom made glass flow cell is mounted on a xyz piezoelectric nanopositioning stage (Mad City Labs) to allow precise displacement. The transmitted beam is then collected by a condenser (Olympus, achromatic/aplanic, NA 0.8) and deflected by a second dichroic mirror toward the detection system. The transmitted power is divided in two by the use of a beam splitter (BS; Thorlabs): half of the beam is sent to a duolateral position sensitive detector with built-in preamplifier (PSD; Pacific Silicon Sensor Incorporated, maximum light intensity $1.5 \mathrm{~W} / \mathrm{cm}^{2}$ ) eventually preceded by a polarizer. When the polarizer is in place the PSD can be used to monitor the linear component of the polarization, thus the particle speed rotation, otherwise it can be used for $\mathrm{X}, \mathrm{Y}$ and $\mathrm{Z}$ positioning detection.

The other half is sent to the torque detection system that is measuring the torque by using two identical PSDs. The imbalance of the two circular components of the polarization, right and left, can be optically measured by translating them into vertical and horizontal components through the use of a quarter waveplate and then by separating them with a PBS. The alignment required for the QWP is at $-45^{\circ}$ respect to the PBS vertical axis. Different filters are set in the photodetector path in order to avoid detector saturation and damage.

A good calibration of the setup is needed in order to translate the measured values in physical quantities. This is discussed in the following paragraph.

\subsection{Torque calibration}

When working with optical tweezers, a precise calibration of the system is necessary in both force and torque measurements in order to obtain quantitative values of force and torque respectively. Three different parameters have to be extracted in each measurement and, in particular, in torque system one should quantify: the torque sensitivity $\beta_{\tau}$ (in $\mathrm{V} / \mathrm{pN} \mathrm{nm}$ ) that allows the conversion of the volt signal in $\mathrm{pN} \mathrm{nm}$, the maximum torque $\tau_{0}$ (in $\mathrm{pN} \mathrm{nm}$ ) and the angular drag coefficient $\gamma$ (in $\mathrm{pN} \mathrm{nm} \mathrm{s).}$
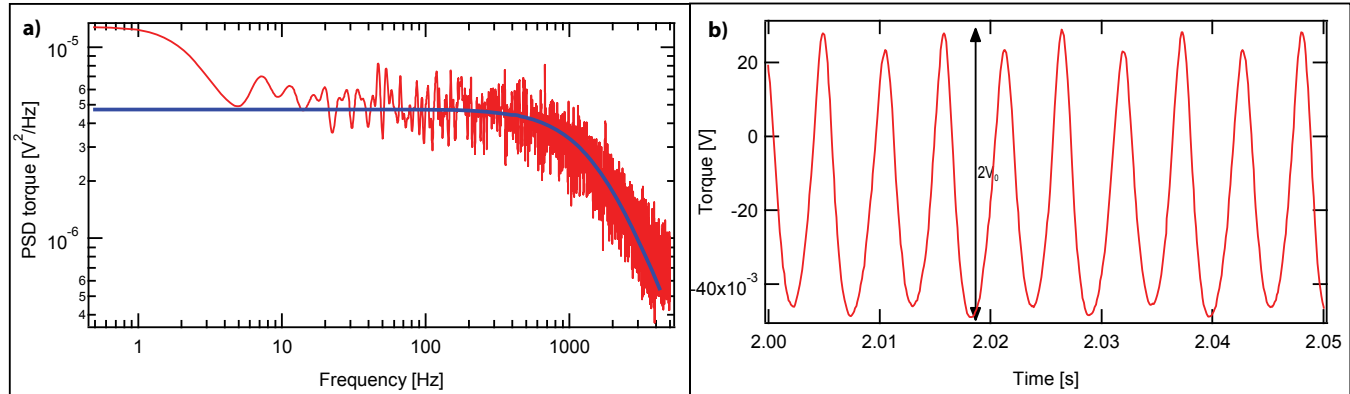

Figure 3. a) Power spectral density of a $\mathrm{NaNbO}_{3}$ nanowire torque measurement taken at polarization rotation $\Omega=0$ i.e. when the particle is not rotating. The extraction of $f_{c}$ and $A_{0}$ is done by fitting the curve with a Lorenztian. b) Torque measurement taken at a polarization rotation $\Omega \neq 0$. The half peak to peak amplitude $V_{0}$ is extracted from it.

Most of the calibration methods available ${ }^{[16,22]}$ are based on extraction of the cut off frequency $f_{c}$ and the zero frequency amplitude $A_{0}$ from the power spectral density of the torque signal taken at $\Omega=0$ (see Figure $3 a$ ). This is possible by fitting the power sprectral density curve with a Lorentzian described as

$$
P_{0}\left(\tau_{m}, f\right)=\frac{A_{0}}{f^{2}+f_{c}^{2}}
$$

A third variable usually extracted is the amplitude $\mathrm{V}_{0}$ of the torque signal experienced by the particle (see Figure 3b). This value can is obtained by a measurement taken at a certain polarization rotation $\Omega \neq 0$. 
Pedaci et al. ${ }^{[22]}$ reported different methods to calibrate an angular trap driven by a rotating linearly polarized beam. In one of the presented methods the calibration parameters are deduced from the measurement of the three independent variables $\mathrm{A}_{0}, \mathrm{f}_{\mathrm{c}}$, and $\mathrm{V}_{0}$ as follows:

$$
\gamma=\frac{4 k_{B} T V_{0}^{2}}{\pi^{2} A_{0}} \quad \tau_{0}=\pi f_{c} \quad \beta_{\tau}=\frac{V_{0}}{\tau_{0}}
$$

The optical torque can though be obtained by dividing the measured torque $\tau_{\mathrm{m}}$ by the torque sensitivity $\beta_{\tau}$ :

$$
\tau=\tau_{m} / \beta_{\tau}
$$

The torque sensitivity allows the conversion of the voltage signals detected by the PSDs in physical units. A method similar to the one presented below, that allows the extraction of the torque sensitivity, has still to be implemented for an angular trap driven by a circularly polarized beam. A proper calibration method would allow the use of rotating nanowires to measure the viscosity of the media in which they are dispersed in. In particular by knowing that $\tau=\gamma \Omega$ and $\gamma=C_{0} \pi \eta L R^{2}\left(1+\delta_{\text {rot }}\right)^{[23]}$ the fluid viscosity $\eta$ of the media can be calculated as:

$$
\eta=\frac{\gamma}{C_{0} \pi L R^{2}\left(1+\delta_{\text {rot }}\right)}=\frac{\tau}{C_{0} \pi L R^{2}\left(1+\delta_{r o t}\right) \Omega}
$$

where $\gamma$ is the drag coefficient, $\mathrm{C}_{0}$ is a constant, $\mathrm{L}$ is the length of the wire, $\mathrm{R}$ is the radius of the wire and $\delta_{\text {rot }}$ is the endeffect correction factor.

\section{EXPERIMENTAL SECTION}

We aimed here to compare the overall performance of the three types of nanowires, i.e. their ability to generate SHG, to waveguide, to be trapped and to be rotated. First we have used a 2-photons confocal microscope (Leica SP5) for high-throughput measurements of the nonlinear optical response. The confocal microscope is based on a LP femtosecond laser pulses centered at $\lambda=854 \mathrm{~nm}$. Nanowires, deposited on a glass slide, were excited and the SHG signal was detected by a photomultiplier. A bandpass filter centered at $427 \mathrm{~nm}$ (with a $10 \mathrm{~nm}$ bandwidth) was placed in front of the detector. The excitation power and all other parameters were kept constant allowing the comparison of the registered signals coming from different samples. More than 20 wires per type have been observed to address the variability of sizes and wire to wire crystallinity dissimilarities. Moreover the averaged SHG signals have been scaled over the wire volume. By comparing the obtained values appears that, as summarized in Table $1, \mathrm{LiNbO}_{3}$ displays the highest nonlinear response while $\mathrm{NaNbO}_{3}$ the smallest ${ }^{[5]}$. Shown data are normalized over the $\mathrm{LiNbO}_{3} \mathrm{SHG}_{\text {average value. }}$

\begin{tabular}{|c|c|c|}
\hline $\mathbf{N a N b O}_{3}$ & $\mathbf{K N b O}_{\mathbf{3}}$ & $\mathbf{L i N b O}_{\mathbf{3}}$ \\
\hline $0.08 \pm 0.03$ (a.u.) & $0.42 \pm 0.15$ (a.u.) & $1.00 \pm 0.28$ (a.u.) \\
\hline
\end{tabular}

Table 1: Average SHG values of the three types of alkaline niobate nanowires normalized over the $\mathrm{LiNbO}_{3}$ one. Reported values are volume scaled.

Subsequently a home-made optical trap has been employed allowing the trapping and the manipulation of nanowires dispersed in DI water. Their capability to waveguide light could be observed by placing the trapped nanowire in a close proximity of the glass coverslip composing the sample chamber (see Figure 4a). In this configuration both nanowire ends are visible and the waveguided SHG signal can be detected. It has been observed that all three types of alkaline niobate nanowires are able to waveguide the SHG signal. This is possible thanks to their high refractive index (from 2.02-2.4) that provides a tight optical confinement. An in-coupling efficiency up to $80 \%$ was measured. Figures $4 \mathrm{~b}, 4 \mathrm{c}$ and $4 \mathrm{~d}$ are showing the waveguided signals for the three samples. 
a)

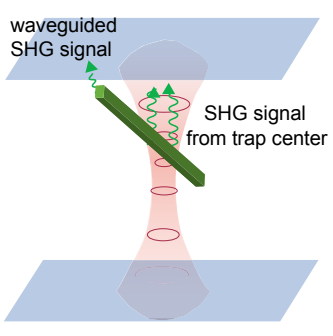

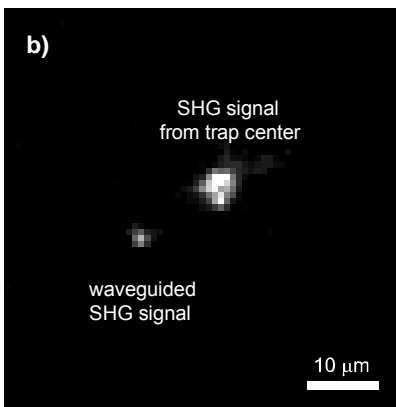
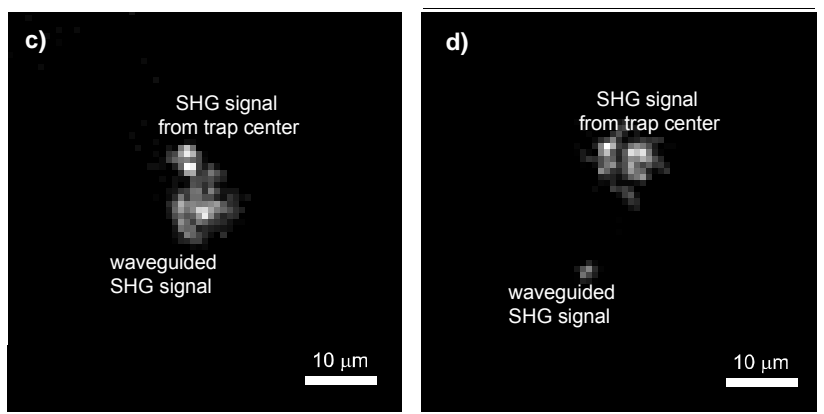

Figure 4: a) Schematics of experimental configuration employed for waveguiding experiments. b) SHG image of $\mathrm{NaNbO}_{3}$ nanowire. c) $\mathrm{SHG}$ image of $\mathrm{KNbO}_{3}$ nanowire. d) $\mathrm{SHG}$ image of $\mathrm{LiNbO}_{3}$ nanowire.

With the same optical setup nanowires can also be rotated by adding a QWP in the beam path right after the power control system: if the angle of the QWP axis is placed at $45^{\circ}$ respect to the beam polarization direction, the beam passing through the QWP is transformed in circularly polarized beam. The angular momentum own by the beam can be transferred to the trapped particle and, thanks to the birefringence of all three types of alkaline niobate nanowires, they can be rotated by the beam. Here we show torque data only for $\mathrm{NaNbO}_{3}$ wires, but also $\mathrm{KNbO}_{3}$ could be rotated. $\mathrm{LiNbO}_{3}$ wires, in principle, can be rotated aswell, however they present a much lower stability in the trap and thus it is difficult to use them for this purpose.

The rotation speed of trapped wires can be obtained by identifying the frequency in the power spectral density (PSD) of the recorded signal for which the amplitude is the highest (see Figure 5a). A precise value of the rotation frequency can be extracted as half of the peak one. A direct proportionality of the rotation speed and the laser power ${ }^{\text {[24- }}$ ${ }^{26]}$ is shown in Figure $5 \mathrm{~b}$ : by increasing the power of the laser beam the wires can be rotated faster. Variation in size of the trapped nanowire changes the moment of inertia and the viscous force in the rotation, affecting the rotation speed. As already observed by SEM and optical microscope, chemical synthesis results in a large variability in sizes of synthesized nanowires and, as a consequence, in a large variability of the rotation speed.

a)

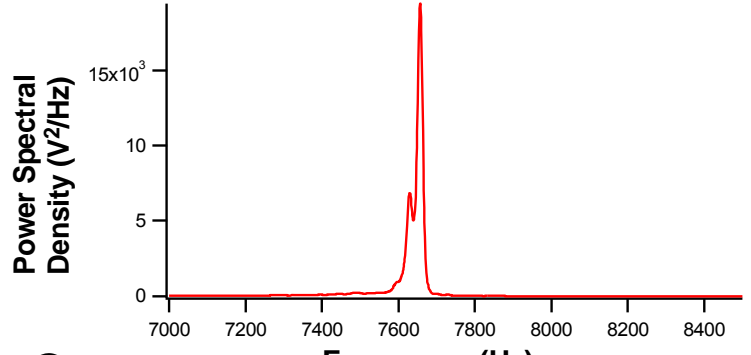

b)

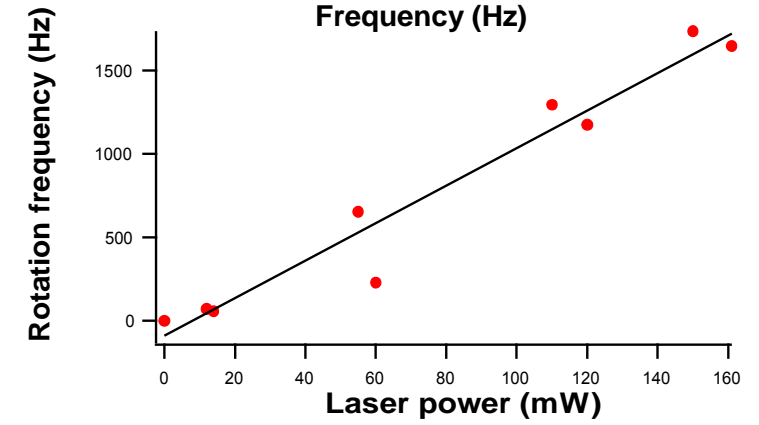

c)

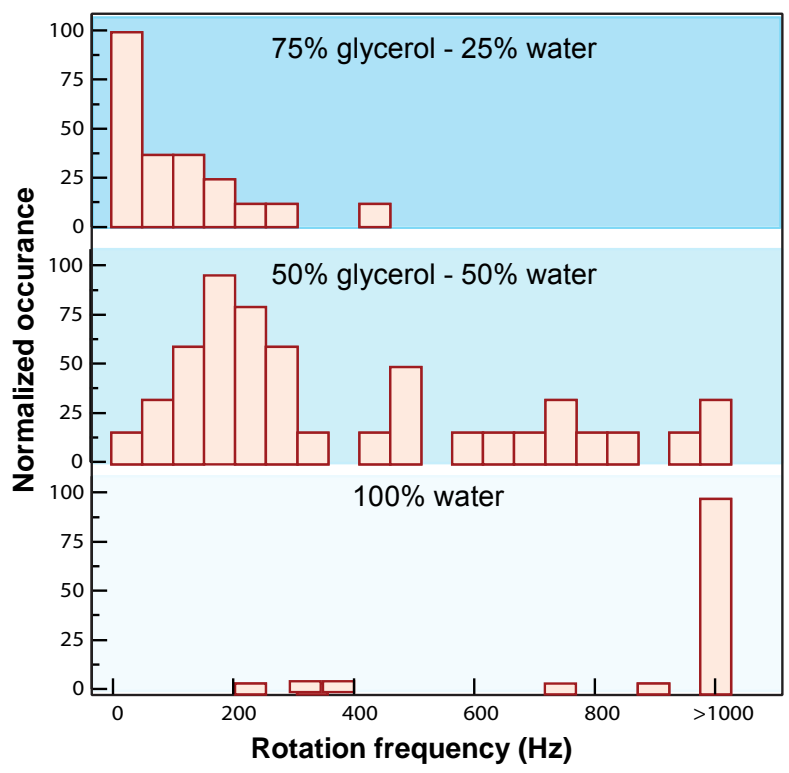

Figure 5: a) Typical peak of power spectral density corresponding to the doubled rotating frequency of the trapped nanowire $2 \Omega=7658 \mathrm{~Hz} \longrightarrow \Omega=3829 \mathrm{~Hz}$. b) Rotation frequency $\Omega$ as a function of laser beam power measured at the focus. c) Rotation frequencies of $\mathrm{NaNbO}_{3}$ nanowires dispersed in different media of various viscosities.

Figure $5 \mathrm{c}$ shows the rotational speed determined from more than $20 \mathrm{NaNbO}_{3}$ wires dispersed in different media, with increasing viscosities (reported in Table 2). 


\begin{tabular}{|c|c|c|}
\hline $\boldsymbol{\eta}_{\text {water }}$ & $\boldsymbol{\eta}_{\mathbf{5 0} / \mathbf{5 0} \text { glycerol } / \text { water }}$ & $\boldsymbol{\eta}_{\mathbf{7 5} / \mathbf{2 5} \text { glycerol } / \text { water }}$ \\
\hline $0.001 \mathrm{~Pa} \mathrm{~s}$ & $0.008 \mathrm{~Pa} \mathrm{~s}$ & $0.055 \mathrm{~Pa} \mathrm{~s}$ \\
\hline
\end{tabular}

Table 2: Theoretically viscosity values for the different employed media.

For low viscosity values, as the water one, more than $70 \%$ of the measured wires are rotating with a rotation speed higher than $1 \mathrm{kHz}$ (up to $5 \mathrm{kHz}$ ). However the range covered by measured rotation speed of different wires is broad, ranging from $10 \mathrm{~Hz}$ to $5 \mathrm{kHz}$. By dispersing wires in a mix composed by $50 \%$ of glycerol and $50 \%$ of water the viscosity is around 8 times larger than the water one (as reported in table 2). Wires are showing a much slower rotation speed with the highest value of $1.5 \mathrm{kHz}$ and $94 \%$ of analyzed wires rotating below $1 \mathrm{kHz}$. We observed a high occurrence of events around $170 \mathrm{~Hz}$. The overall range is more than 3 times smaller than the one in the previous case. Considering a media composed by $75 \%$ of glycerol and $25 \%$ of water the expected viscosity is around 50 times higher than the water one (data reported in table 2). Wires are rotating much slower than in previous cases having a maximum rotation speed of $430 \mathrm{~Hz}$. $90 \%$ of wires rotate slower than $250 \mathrm{~Hz}$ with a peak of occurrence around $60 \mathrm{~Hz}$.

The variability in rotation speed is very pronounced for low viscosity media, while is decreasing by increasing the viscosity. It is then clear that by increasing the viscosity of the medium, the influence of the wires size variability decreases. This kind of rotating structures could thus be used to measure the viscosity of the media, from the torque measurement, just for high viscous media.

Finally to allow the use of alkaline niobate nanowires in biomedical imaging and as opto-mechanical probes a biomolecular functionalization is necessary. The glass slide functionalization process described by Tanner et al. ${ }^{[27]}$ has been adapted to the chemically synthesized nanowires and successfully tested over the three types of wires (data not shown). By coating the wires with APTES (3-aminopropyltriethoxysilane), biotinylated PEG (polyethylene glycol) and finally streptavidin, wires are biocompatible and ready for an eventual bind with biotin. By employing a biotinylated dsDNA intercalated with YOYO dyes, it was possible to check with a fluorescent microscope that the functionalization process worked and that wires are covered more or less uniformly by a streptavidin layer.

\section{CONCLUSION}

In summary, we report several interesting points: the comparative study of $\mathrm{SH}$ signal in $\mathrm{XNbO}_{3}$ nanowires establish that the largest nonlinear response is the $\mathrm{LiNbO}_{3}$ one. We showed the efficient waveguiding of $\mathrm{SH}$ signal through nanowire, prerequisite for a future use of the $\mathrm{XNbO}_{3}$ nanowires as local noninvasive light sources into biological cells or tissues. Nanowires can be rotated at different speed, and eventually used for coarse viscosity measurement. Functionalization tests were performed and a successful process to functionalize niobate nanowires was developed allowing the employment of them in bio applications. This is a prerequisite for the use of these probes for torque measurement of double strand DNA typically performed by magnetic tweezers ${ }^{[28]}$.

In conclusion we can associate the following application to the different types of wires: $\mathrm{LiNbO}_{3}$ nanowires display the highest nonlinear response thus they are best suited for the use as markers in imaging. On the other hand, their trapping and rotational stability is much worse compared to the other two types of alkaline niobate nanowires, therefore $\mathrm{KNbO}_{3}$ or $\mathrm{NaNbO}_{3}$ nanowires are good candidates as opto-mechanical probes while $\mathrm{LiNbO}_{3}$ ones are not. As all three types of $\mathrm{XNbO}_{3}$ nanowires display similar in-coupling wave-guiding efficiency, as potential waveguides, we would recommend $\mathrm{NaNbO}_{3}$ nanowires due to their highest aspect ratio.

\section{ACKNOWLEDGEMENT}

This work was supported by the Swiss National Science Foundation (FNS) with Grants 200021-125319 and CRSII3_132396. We thank the Centre Interdisciplinaire de Microscopic Electronique (CIME) at EPFL for access to electron microscopes and Bioimaging and Optics Platform (PTBIOP) for access to confocal microscope. A particular thank to Dr. F. Pedaci for the useful discussions. 


\section{REFERENCES}

1. Arizmendi, L., Photonic applications of lithium niobate crystals. Physica Status Solidi a-Applied Research, 2004. 201(2): p. 253-283.

2. Shi, H.F., et al., NaNbO3 Nanostructures: Facile Synthesis, Characterization, and Their Photocatalytic Properties. Catalysis Letters, 2009. 132(1-2): p. 205-212.

3. Zhu, H.Y., et al., Structural evolution in a hydrothermal reaction between Nb2O5 and NaOH solution: From $\mathrm{Nb} 2 \mathrm{O} 5$ grains to microporous $\mathrm{Na} 2 \mathrm{Nb} 2 \mathrm{O} 6$ center $\operatorname{dot}(2) / 3 \mathrm{H} 2 \mathrm{O}$ fibers and $\mathrm{NaNbO} 3$ cubes. Journal of the American Chemical Society, 2006. 128(7): p. 2373-2384.

4. Li, L.H., et al., Wire Structure and Morphology Transformation of Niobium Oxide and Niobates by Molten Salt Synthesis. Chemistry of Materials, 2009. 21(7): p. 1207-1213.

5. $\quad$ Dutto, F., et al., Nonlinear Optical Response in Single Alkaline Niobate Nanowires. Nano Letters, 2011. 11(6): p. 2517-2521.

6. Wu, S.Y., X.Q. Liu, and X.M. Chen, Hydrothermal synthesis of NaNbO3 with low NaOH concentration. Ceramics International, 2010. 36(3): p. 871-877.

7. Santulli, A.C., et al., Synthesis of single-crystalline one-dimensional LiNbO3 nanowires. Crystengcomm, 2010. 12(10): p. 2675-2678.

8. $\quad$ Boyd, R.W., Nonlinear optics. 3rd ed. 2008, Amsterdam ; Boston: Academic Press. xix, 613 p.

9. $\quad$ Franken, P.A., et al., Generation of Optical Harmonics. Physical Review Letters, 1961. 7(4): p. 118-\&.

10. Perkins, T.T., Optical traps for single molecule biophysics: a primer. Laser \& Photonics Reviews, 2009. 3(1-2): p. 203-220.

11. Parkin, S., et al., Optical torque on microscopic objects. Laser Manipulation of Cells and Tissues, 2007. 82: p. 525-561.

12. Bishop, A.I., et al., Optical application and measurement of torque on microparticles of isotropic nonabsorbing material. Physical Review A, 2003. 68(3).

13. Bishop, A.I., et al., Optical microrheology using rotating laser-trapped particles. Physical Review Letters, 2004. 92(19).

14. Dekker, N.H., et al., Electron Beam Fabrication of Birefringent Microcylinders. Acs Nano, 2011. 5(2): p. 14181427.

15. Deufel, C., et al., Nanofabricated quartz cylinders for angular trapping: DNA supercoiling torque detection. Nature Methods, 2007. 4(3): p. 223-225.

16. Gutierrez-Medina, B., et al., An Optical Apparatus for Rotation and Trapping. Methods in Enzymology, Vol 475: Single Molecule Tools, Pt B, 2010. 474: p. 377-404.

17. La Porta, A. and M.D. Wang, Optical torque wrench: Angular trapping, rotation, and torque detection of quartz microparticles. Physical Review Letters, 2004. 92(19).

18. Neuman, K.C., Single-molecule Measurements of DNA Topology and Topoisomerases. Journal of Biological Chemistry, 2010. 285(25): p. 18967-18971.

19. Pedaci, F., et al., Excitable particles in an optical torque wrench. Nature Physics, 2011. 7(3): p. $259-264$.

20. Wang, G.Z., et al., Hydrothermal synthesis and characterization of KNbO3 nanorods. Crystengcomm, 2009. 11(9): p. 1958-1963.

21. Wang, G.Z., et al., Synthesis of KNbO3 Nanorods by Hydrothermal Method. Journal of Nanoscience and Nanotechnology, 2009. 9(2): p. 1465-1469.

22. Pedaci, F., et al., Calibration of the optical torque wrench. Optics Express, 2012. 20(4): p. 3787-3802.

23. Tirado, M.M. and J. Garciadelatorre, Rotational-Dynamics of Rigid, Symmetric Top Macromolecules Application to Circular-Cylinders. Journal of Chemical Physics, 1980. 73(4): p. 1986-1993.

24. Wood, T.A., et al., Characterization of microparticles with driven optical tweezersw. Faraday Discussions, 2008. 137: p. 319-333.

25. Sun, X.D., et al., Optical rotation and manipulation of micro-sized LiNbO3 crystals and single-walled carbon nanotubes bundles. Colloids and Surfaces a-Physicochemical and Engineering Aspects, 2008. 313: p. 488-491.

26. Neves, A.A.R., et al., Rotational dynamics of optically trapped nanofibers. Optics Express, 2010. 18(2): p. 822830.

27. Tanner, N.A. and A.M. van Oijen, Visualizing DNA Replication at the Single-Molecule Level. Methods in Enzymology, Vol 475: Single Molecule Tools, Pt B, 2010. 474: p. 259-278.

28. Lipfert, J., et al., Magnetic torque tweezers: measuring torsional stiffness in DNA and RecA-DNA filaments. Nature Methods, 2010. 7(12): p. 977-U54. 